\title{
Sub-Optimal CD4 T-Lymphocyte Responses among HIV Infected Patients who Develop TB during the First Year of ART Ingrid Eshun-Wilson ${ }^{1,2 *}$, Jantjie $\mathrm{J}$ Taljaard ${ }^{3}$ and Jean B Nachega ${ }^{1,4}$
}

${ }^{1}$ Centre for Infectious Diseases, Stellenbosch University, Parow, 7505, Cape Town South Africa

${ }^{2}$ Department of Epidemiology and Biostatistics, University of San Francisco, California, 41097, USA

${ }^{3}$ Centre for Infectious Diseases and Division of Internal Medicine, Stellenbosch University, Parow, 7505, Cape Town South Africa

${ }^{4}$ John Hopkins School of Public Health, Johns Hopkins University, Baltimore, Maryland, USA

\begin{abstract}
Study background: Poor CD4 T-lymphocyte responses to anti-retroviral treatment (ART) are associated with increased HIV disease progression and mortality. In sub-Saharan Africa a substantial proportion of HIV infected patients are co-infected with TB. This study evaluated the effect of active TB presenting after ART initiation on immunological responses to ART.
\end{abstract}

Methods: A retrospective cohort study was conducted of patients initiated on ART in a South African academic hospital between 1 January 2004 and 15 May 2008. Changes in CD4 T-lymphocyte count, virological suppression and incident TB episodes occurring in the first year of ART were assessed. Sub-optimal CD4 responses were defined as 'failure to increase CD4 T-lymphocyte count by $50 \mathrm{cells} / \mu \mathrm{l}$ at 6 month on ART'.

Results: The cohort for analysis included 691 patients. $141(20.4 \%)$ had sub-optimal CD4 responses at 6 months on ART. 49 patients (7.1\%) developed incident TB within the first 12 months of ART. After adjustment for age, sex, baseline CD4 count and detectable viral load, patients with incident TB were found to have a 2.20 times greater odds of a sub-optimal CD4 response at 6 month of ART as compared to those who were TB free $(95 \% \mathrm{Cl}: 1.14-4.23)$.

Conclusion: Incident TB was associated with a poor CD4 response during early ART in this cohort. Although the direction of causality cannot be determined from these data, these findings provide additional support for the initiation of ART at higher CD4 counts.

Keywords: Sub-optimal; CD4; TB; ART

\section{Introduction}

Sub-optimal CD4 T-lymphocyte responses to anti-retroviral treatment (ART) occur in $15 \%$ to $21 \%$ of patients during the first year of treatment [1-4]. Risk factors which have been repeatedly associated with sub-optimal responses include; older age and baseline CD4 T-cell count [2-8]. Sub-optimal CD4 responses are associated within increased progression of HIV disease and higher AIDS related mortality $[1,3,7,8]$.

In South African cohorts HIV infection is complicated by high rates of TB co-infection; during the first year of ART, TB incidence is reported to be 3.5 cases per 100 person-years. This decreases substantially with increased time on ART and at 5 years; incidence rates reduce to 1.01 cases per 100 person-years [9]. Studies evaluating immunological responses to ART have found poor responses to occur in patients who develop incident TB after initiating ART, these studies have however been limited by a small numbers of incident TB cases or a lack of corresponding viral load measurements $[9,10]$. Further studies which evaluated the combined effect of TB prior to ART, at baseline and after ART initiation have found no association with CD4 T-lymphocyte count or virological response $[11,12]$.

The aim of this study was to determine the association between incident TB occurring during the first year after ART initiation, and sub-optimal immunological responses to ART. It was hypothesized that HIV infected patients who were receiving ART and developed TB during the first year of ART would have poorer CD4 responses as compared to those who did not develop incident TB.

\section{Materials and Methods}

\section{Study design and study population}

A retrospective cohort study was conducted of patients initiating
ART through the national ART program at Tygerberg Academic Hospital Infectious Diseases Clinic, in Cape Town, South Africa between 1 January 2004 and 15 May 2008. Patients initiating ART received a backbone of stavudine or zidovudine with lamivudine and either efavirenz or nevirapine. Patients with TB were preferentially initiated or switched to an efavirenz containing regimen. Follow-up data was censored on 28 Feb 2009. TB treatment was Rifampicin based and routinely provided for 6-8 months according to WHO guidelines [13] All patients initiating ART were screened for active TB through clinical evaluation and microbiological tests and chest-radiograph. Viral load and CD4 T-lymphocyte count measurements were performed routinely every 6 months after initiating ART. If clinical failure was suspected viral load measurements were performed outside of the 6 month window at the physicians' discretion. Baseline viral load measurements were only available between 2004 and 2005 due to changes in national policy after this period. In an attempt to decentralize care a large proportion of patients were transferred to peripheral community ART clinics after initiating ART diminishing the number of patients with follow-up beyond the first 6 months of ART.

*Corresponding authors: Ingrid Eshun-Wilson, Centre for Infectious Diseases, Stellenbosch University, Parow, 7505, Cape Town, South Africa, Tel: +1 4159871439; E-mail: wilson.ingrid@gmail.com

Received September 06, 2011; Accepted January 25, 2012; Published January 29,2012

Citation: Eshun-Wilson I, Taljaard JJ, Nachega JB (2012) Sub-Optimal CD4 T-Lymphocyte Responses among HIV Infected Patients who Develop TB during the First Year of ART. J AIDS Clinic Res 3:135. doi:10.4172/2155-6113.1000135

Copyright: @ 2012 Eshun-Wilson I, et al. This is an open-access article distributed under the terms of the Creative Commons Attribution License, which permits unrestricted use, distribution, and reproduction in any medium, provided the original author and source are credited. 
Citation: Eshun-Wilson I, Taljaard JJ, Nachega JB (2012) Sub-Optimal CD4 T-Lymphocyte Responses among HIV Infected Patients who Develop TB during the First Year of ART. J AIDS Clinic Res 3:135. doi:10.4172/2155-6113.1000135

Page 2 of 4

\section{Definitions and laboratory measurements}

Baseline measurements (CD4 T-lymphocyte count and viral load) were included if performed within six months prior to ART initiation and once on treatment follow up measurements were included if performed within 2 months (+/-) of the 6 or 12 month time points.

The diagnosis of active TB was made both at peripheral TB clinics and at the study site with the use of World Health Organisation (WHO) TB case definitions [14]. These diagnoses were based on clinical, microbiological and radiological findings. Data from peripheral clinic $\mathrm{TB}$ record cards were routinely captured in the clinic database if patients developed TB while on ART. 'Incident TB' was defined as active TB which developed after initiating ART and 'prevalent TB' as active TB for which treatment was initiated prior to ART.

A sub-optimal CD4 response at 6 months was defined as a failure of the CD4 T-lymphocyte count to rise from baseline by more than 50 cells/ $\mu \mathrm{l}[2,3,8]$. A detectable viral load during the first year was determined as any viral load measurement above 400 copies/ml between 6 and 12 months on ART.

\section{Sample size estimation}

All available cohort data was used for evaluation in this study.

\section{Data sources and ethics approval}

Data was abstracted from clinical records and clinic and laboratory databases. The Health Research Ethics Review Committee of the University of Stellenbosch approved the protocol.

\section{Statistical analysis}

Stata version 11 was used for data management and analysis. Logistic regression was used for all univariable and multivariable analyses. The results are presented as odds ratios (OR) with $95 \%$ confidence intervals (CI). All significance test results represent likelihood ratio tests and are two-sided $(\alpha=0.05)$. Multivariable models were constructed using a forward step-wise approach. Age and sex were included a-priori in models. Interactions between terms were evaluated for each model.

\section{Results}

Of 936 patients who had received ART for 6 months or longer, 691 were eligible for inclusion into the study (Figure 1) with a total of 660 patient years of follow up.

When excluded cases were compared to those included in the final cohort there was no difference in age (median 34.3yrs vs 35.5yrs;

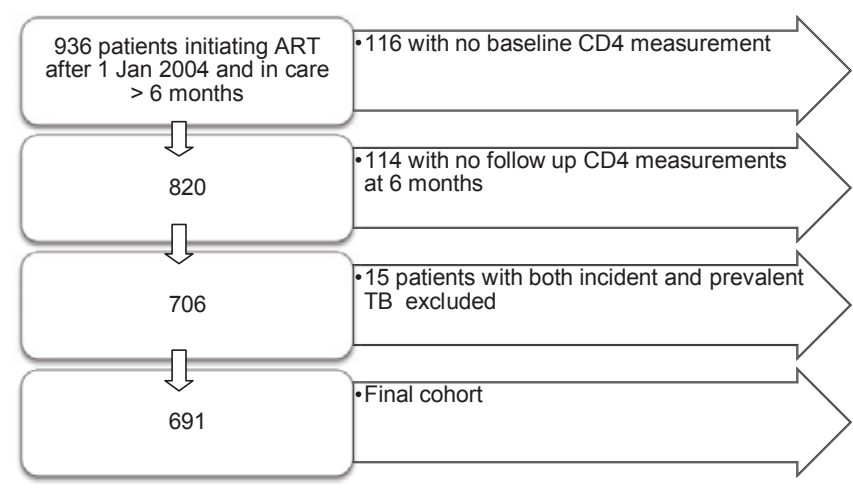

Figure 1: Cohort construction. $\mathrm{p}=0.326$ ), sex (female $=31.4 \%$ vs $34 \%$; $\mathrm{p}=0.460$ ) or occurrence of incident TB ( $8.9 \%$ vs $7.1 \%$; $\mathrm{p}=0.346)$.

The median age of the final cohort was 35 years and $66 \%$ of the cohort was female (Table 1). Incident TB developed in 49 (7.1\%) of patients within the first 12 months of ART, with an incidence rate of 7.4 cases per 100 patient years of follow up. The median timing of incident TB during the first year of ART was 4.24 months (IQR: 1.8-6.9 months). 179 patients were already receiving TB treatment (prevalent TB) for a median of 3.2 months (IQR: 2.03-4.83 months) at the time of ART initiation.

Sub-optimal CD4 T-cell responses at 6 months on ART were seen in $141(20.4 \%)$ of patients in the cohort. A detectable viral load between 6 and 12 months after initiating ART occurred in 72 (10.42\%) patients and among these the median detectable viral load was 5.21 copies $/ \mathrm{ml}$ (IQR: 4.63-5.81 copies/ml).

Among patients who developed incident TB within the $1^{\text {st }}$ year of ART, 17 of 49 (34.7\%) had sub-optimal CD4 T-cell responses as compared to 104 of 478 (21.8\%) patients who had no evidence of TB.

The univariable and multivariable analyses of the association between incident TB and sub-optimal CD4 responses (Table 2) were restricted to those who did not have prevalent TB at baseline $(n=527)$. After adjustment for age, sex, baseline CD4 count and a detectable viral load during first year of ART, patients who developed incident TB were

\begin{tabular}{|c|c|c|}
\hline \multicolumn{3}{|l|}{ Demographic characteristics } \\
\hline Age [median (IQR) - years] & 35 & $(30-42)$ \\
\hline Sex - Female [n (\%)] & 456 & $(66.0 \%)$ \\
\hline Baseline CD4 count [median (IQR) - cells/ $\mu \mathrm{l}]$ & 122 & $(59-173)$ \\
\hline Baseline viral load [median (IQR) - log copies/ml] ${ }^{\star}$ & 5.35 & $(4.86-5.81)$ \\
\hline \multicolumn{3}{|l|}{ TB history } \\
\hline Incident TB during $1^{\text {st }} 12$ months of ART [n (\%)] & 49 & (7.1) \\
\hline Prevalent TB at ART initiation [n (\%)] & 179 & $(25.9)$ \\
\hline \multicolumn{3}{|l|}{ Follow -up data } \\
\hline Sub-optimal CD4 responses at 6 months $[\mathrm{n}(\%)]^{\dagger}$ & 141 & $(20.4)$ \\
\hline Detectable viral load within $1^{\text {st }} 12$ months of ART $[n(\%)]^{\ddagger}$ & 72 & $(10.4)$ \\
\hline \multicolumn{3}{|l|}{ * Baseline viral load only available for 473 patients } \\
\hline \multicolumn{3}{|c|}{$\dagger$ Failure to increase $C D 4$ count by $\geq 50$ cells $/ \mu$ at 6 months on ART } \\
\hline
\end{tabular}

Table 1: Descriptive characteristics of cohort $(n=691)$.

\begin{tabular}{|c|c|c|c|c|c|c|c|}
\hline \multicolumn{2}{|l|}{ Variable } & \multicolumn{3}{|c|}{ Univariable analysis } & \multicolumn{3}{|c|}{ Multivariable analysis ** } \\
\hline & & OR & $95 \% \mathrm{Cl}$ & $p$-value & OR & $95 \% \mathrm{Cl}$ & p-value \\
\hline \multirow[t]{2}{*}{ Incident TB } & No & 1 & & \multirow{2}{*}{0.050} & 1 & & \multirow{2}{*}{0.021} \\
\hline & Yes & 1.91 & $1.02-3.58$ & & 2.20 & $1.14-4.23$ & \\
\hline \multicolumn{2}{|l|}{ Age } & 1.05 & $1.02-1.07$ & $<0.001$ & 1.05 & $1.02-1.07$ & $<0.001$ \\
\hline \multirow[t]{2}{*}{ Sex } & Male & 1 & & \multirow{2}{*}{0.162} & 1 & & \multirow{2}{*}{0.476} \\
\hline & Female & 0.74 & $0.48-1.12$ & & 0.84 & $0.52-1.36$ & \\
\hline \multicolumn{2}{|c|}{ Baseline CD4 count } & 1.01 & $1.00-1.01$ & $<0.001$ & 1.01 & $1.004-1.01$ & $<0.001$ \\
\hline \multirow{2}{*}{$\begin{array}{l}\text { Detectable } \\
\text { viral load † }\end{array}$} & No & 1 & & \multirow{2}{*}{0.231} & 1 & & \multirow{2}{*}{0.046} \\
\hline & Yes & 1.48 & $0.79-2.75$ & & 2.01 & 1.03-3.9 & \\
\hline
\end{tabular}

${ }^{*}$ Analysis restricted to the 527 patients with no TB at baseline

${ }^{* *}$ No interactions detected between variables.

† Detectable viral load between 6 and12 months on ART

Table 2: Factors associated with sub-optimal cd4 response at 6 months $(n=527)^{*}$. 
found have a 2.2 times greater odds of a sub-optimal CD4 response at 6 months on ART (95\% CI: 1.14-4.23; p-value: 0.021).

\section{Discussion}

In this cohort, patients who developed incident $\mathrm{TB}$ during the first year after initiating ART were more likely to have suboptimal CD4 responses at 6 months of treatment. It is however not possible to determine the causal direction of this relationship from such observational data. Active TB has been shown to cause CD4 lymphopaenia independently of HIV status this may have contributed to the poor immunological responses seen during active TB infection in this cohort [15].

In a similar Ugandan cohort of $5982 \mathrm{HIV}$ infected patients initiated on ART, incident TB was found to occur in $5.6 \%$ of patients within 12 months and was associated with sub-optimal CD4 responses at up to 24 months [10]. This suggests that incident TB in HIV infected patients is associated with long lasting immune suppression, virological suppression and adherence data were however not available for analysis in this study. A South African study evaluating 27 incident TB cases occurring over 5 years found sub-optimal CD4 responses to be associated with incident TB independently of virological failure [9].

Strength of our study is the availability of viral load data after ART initiation and substantially more cases of incident TB as compared to those evaluated by Lawn et al. [9]. The ability to adjust for elevated viral load in the multivariable analysis compensates to some degree for lack of direct data on ART adherence in this study. The high viral load measurements seen in patients with a detectable viral load suggest poor ART adherence or treatment failure in these cases. Direct adherence data would however have contributed further to this analysis and reduced residual confounding.

The association between age and sub-optimal CD4 recovery has been attributed to the deteriorating thymic function which occurs with advancing age and lower naive/memory $\mathrm{CD} 4$ ratios among older patients $[16,17]$. The need to re-establish immune function more rapidly during early ART among severely immunocompromised patients may explain the better short term CD4 responses among patients with low baseline CD4 count [5]. Patients with low CD4 T-lymphocyte count at baseline have however been shown to have poorer long term CD4 responses and do not reach the same $\mathrm{CD} 4$ plateau over time as compared to those with higher CD4 T-lymphocyte counts at baseline [6,7].

The proportion of patients with either missing baseline or followup CD4 measurements limited the number of observations available for analysis at the 6 month time point and could have introduced bias into the study findings. Demographic characteristics and occurrence of TB of these excluded patients did however not differ from the remaining cohort. A further study limitation was the lack of information on exact ART treatment regimen at the time of TB treatment initiation. This would have helped in the evaluation of whether a time lag between starting TB treatment and switching from NVP to EFV impacted on virological responses. A longer duration of follow-up could have helped to ascertain whether early sub-optimal responses in patients with incident TB persist beyond the first year of ART. Other unmeasured confounders such as co-morbidities and other opportunistic infections may have impacted on $\mathrm{CD} 4$ responses in this cohort.

In this setting where ART is initiated at an advanced stage of immunosuppression, incident $\mathrm{TB}$ is associated with poor $\mathrm{CD} 4$ T-lymphocyte responses. Although the direction of causality cannot be determined from these data, these findings provide additional support for the initiation of ART at higher CD4 counts where the underlying risk of developing active TB is lower and where long term immune recovery is improved.

\section{Acknowledgements}

Marina La Grange and Dr M D Zeier for assistance with data base management. Dr C Godwin and Dr J Gorga for assistance with data entry.

Dr I Eshun-Wilson is a recipient of the NIH-Fogarty International ICORHTA and South African TB/AIDS Research Training (SATBAT) Grant Fellowship, 5U2RTWOO7370.

$\mathrm{Dr}$ JB Nachega is the recipient of a United States NIAID/NIH Mentored PatientOriented Research Career Award K23 Al068582-01 and European Developing Countries Clinical Trial Partnership Senior Fellowship TA-08-40200-021.

\section{References}

1. Grabar S, Le Moing V, Goujard C, Leport C, Kazatchkine MD, et al. (2000) Clinical outcome of patients with HIV-1 infection according to immunologic and virologic response after 6 months of highly active antiretroviral therapy. Ann Intern Med 133: 401-410.

2. Nakanjako D, Kiragga A, Ibrahim F, Castelnuovo B, Kamya MR, et al. (2008) Sub-optimal CD4 reconstitution despite viral suppression in an urban cohort on antiretroviral therapy (ART) in sub-Saharan Africa: frequency and clinical significance. AIDS Res Ther 5: 23.

3. Tuboi SH, Brinkhof MW, Egger M, Stone RA, Braitstein P, et al. (2007) Discordant responses to potent antiretroviral treatment in previously naive HIV-1-infected adults initiating treatment in resource-constrained countries: the antiretroviral therapy in low-income countries (ART-LINC) collaboration. J Acquir Immune Defic Syndr 45: 52-59.

4. Marimoutou C, Chene G, Mercie P, Neau D, Farbos S, et al. (2001) Prognostic factors of combined viral load and CD4+ cell count responses under triple antiretroviral therapy, Aquitaine cohort, 1996-1998. J Acquir Immune Defic Syndr 27: 161-167.

5. Lawn SD, Myer L, Bekker LG, Wood R (2006) CD4 cell count recovery among HIV-infected patients with very advanced immunodeficiency commencing antiretroviral treatment in sub-Saharan Africa. BMC Infect Dis 6: 59.

6. Moore RD, Keruly JC (2007) CD4+ cell count 6 years after commencement of highly active antiretroviral therapy in persons with sustained virologic suppression. Clin Infect Dis 44: 441-446.

7. Garcia F, de Lazzari E, Plana M, Castro P, Mestre G, et al. (2004) Longterm CD4+ T-cell response to highly active antiretroviral therapy according to baseline CD4+ T-cell count. J Acquir Immune Defic Syndr 36: 702-713.

8. Moore DM, Hogg RS, Yip B, Wood E, Tyndall M, et al. (2005) Discordant immunologic and virologic responses to highly active antiretroviral therapy are associated with increased mortality and poor adherence to therapy. J Acquir Immune Defic Syndr 40: 288-293

9. Lawn SD, Badri M, Wood R (2005) Tuberculosis among HIV-infected patients receiving HAART: long term incidence and risk factors in a South African cohort. AIDS 19: 2109-2116.

10. Hermans SM, Kiragga AN, Schaefer P, Kambugu A, Hoepelman Al, et al. (2010) Incident tuberculosis during antiretroviral therapy contributes to suboptimal immune reconstitution in a large urban HIV clinic in sub-Saharan Africa. PLoS One 5: e10527.

11. Lawn SD, Myer L, Bekker LG, Wood R (2006) Burden of tuberculosis in an antiretroviral treatment programme in sub-Saharan Africa: impact on treatment outcomes and implications for tuberculosis control. AIDS 20: 1605-1612.

12. Breen RA, Miller RF, Gorsuch T, Smith CJ, Ainsworth J, et al. (2006) Virologica response to highly active antiretroviral therapy is unaffected by antituberculosis therapy. J Infect Dis 193: 1437-1440.

13. World Health Organisation (2009) Treatment of tuberculosis guidelines. 
Citation: Eshun-Wilson I, Taljaard JJ, Nachega JB (2012) Sub-Optimal CD4 T-Lymphocyte Responses among HIV Infected Patients who Develop TB during the First Year of ART. J AIDS Clinic Res 3:135. doi:10.4172/2155-6113.1000135

Page 4 of 4

14. World Health Organisation (2007) Tuberculosis Global Report. Definitions of Tuberculosis Cases and Treatment Outcomes.

15. Kony SJ, Hane AA, Larouze B, Samb A, Cissoko S, et al. (2000) Tuberculosisassociated severe CD4+ T-lymphocytopenia in HIV-seronegative patients from Dakar. SIDAK Research Group. J Infect 41: 167-171.

16. Gutierrez F, Padilla S, Masia M, Iribarren JA, Moreno S, et al. (2008) Patients' characteristics and clinical implications of suboptimal CD4 T-cell gains after 1 year of successful antiretroviral therapy. Curr HIV Res 6: 100-107.

17. Gandhi RT, Spritzler J, Chan E, Asmuth DM, Rodriguez B, et al. (2006) Effect of baseline- and treatment-related factors on immunologic recovery after initiation of antiretroviral therapy in HIV-1-positive subjects: results from ACTG 384 . J Acquir Immune Defic Syndr 42: 426-434. 